\title{
SEVERAL INTEGRAL REPRESENTATIONS INVOLVING TRIPLE HYPERGEOMETRIC FUNCTIONS
}

\author{
Junesang Choi, Anvar Hasanov and Mamasali Turaev
}

\begin{abstract}
A (presumably) new class of generalized triple hypergeometric functions is presented. We also give integral representations of Laplace type for certain special cases of the new class of functions.
\end{abstract}

\section{Introduction and Preliminaries}

Investigation of multiple hypergeometric functions is essentially motivated by the fact that the solutions of many applied problems, for example, the thermal conductivity and dynamics, electromagnetic oscillation and aerodynamics, quantum mechanics and potential theory, are obtainable with the help of such hypergeometric (higher and special or transcendent) functions $[1,4,5,6]$. Functions of such kind are often referred to as special functions of mathematical physics. They mainly appear in the solution of partial differential equations which are dealt with harmonic analysis method (see [2]). In view of various applications, it is interesting in itself and seems to be very important to make a continuous research of multiple hypergeometric functions. For instance, in [10], a comprehensive list of hypergeometric functions of three variables as many as 205 is recorded, together with their regions of convergence. In the course of investigation of properties of several classes of triple hypergeometric functions of Exton type $X_{1}, \ldots, X_{20}$ [3] and Srivastava's hypergeometric functions $H_{A}, H_{B}, H_{C}$, and $F^{(3)}[7,8,10]$, we have encountered a number of generalized triple hypergeometric functions of the fourth order whose series representations involve such products as $\left(a_{j}\right)_{2 m+2 n+p},\left(g_{j}\right)_{m+n}$ and $\left(e_{j}\right)_{m+n+p}$, and various combinations of

Received January 31, 2011. Accepted February 25, 2011.

2000 Mathematics Subject Classification. 33C20, 44A45.

Key words and phrases. Triple hypergeometric series; Pochhammer symbol; Exton type hypergeometric functions, the Srivastava's hypergeometric functions. 
indices. This investigation enables us to present the following (presumably) new class of generalizations of the Exton type functions:

$$
\begin{aligned}
& \mathcal{G}^{(3)}[x, y, z]
\end{aligned}
$$

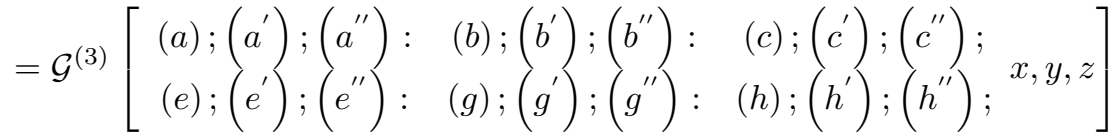

$$
\begin{aligned}
& =\sum_{m, n, p=0}^{\infty} \Lambda(m, n, p) \frac{x^{m}}{m !} \frac{y^{n}}{n !} \frac{z^{p}}{p !},
\end{aligned}
$$

where

$$
\begin{gathered}
\Lambda(m, n, p)=\frac{\prod_{j=1}^{A}\left(a_{j}\right)_{2 m+2 n+p} \prod_{j=1}^{A^{\prime}}\left(a_{j}^{\prime}\right)_{2 m+n} \prod_{j=1}^{A^{\prime \prime}}\left(a_{j}^{\prime \prime}\right)_{n+2 p}}{\prod_{j=1}^{E}\left(e_{j}\right)_{m+n+p} \prod_{j=1}^{E^{\prime}}\left(e_{j}^{\prime}\right)_{2 m+n} \prod_{j=1}^{E^{\prime \prime}}\left(e_{j}^{\prime \prime}\right)_{n+2 p}} \\
\frac{\prod_{j=1}^{B}\left(b_{j}\right)_{m+n} \prod_{j=1}^{B^{\prime}}\left(b_{j}^{\prime}\right)_{n+p} \prod_{j=1}^{B^{\prime \prime}}\left(b_{j}^{\prime \prime}\right)_{m+p}}{\prod_{j=1}^{G}\left(g_{j}\right)_{m+n} \prod_{j=1}^{G^{\prime}}\left(g_{j}^{\prime}\right)_{n+p} \prod_{j=1}^{G^{\prime \prime}}\left(g_{j}^{\prime \prime}\right)_{m+p}} \cdot \frac{\prod_{j=1}^{C}\left(c_{j}\right)_{m} \prod_{j=1}^{C^{\prime}}\left(c_{j}^{\prime}\right)_{n} \prod_{j=1}^{C^{\prime \prime}}\left(c_{j}\right)_{m} \prod_{j=1}^{H^{\prime}}\left(h_{j}^{\prime}\right)_{n} \prod_{j=1}^{H^{\prime \prime}}\left(h_{j}^{\prime \prime}\right)_{p}}{\prod_{j=1}},
\end{gathered}
$$

where $(a)$ abbreviates the array of A parameters $a_{1}, \ldots, a_{A}$, with similar interpretations for $b, b^{\prime}, b^{\prime \prime}$, and so on. We present various representations for triple hypergeometric functions of fourth order of special cases of (1.1):

$$
\begin{aligned}
& \mathcal{G}_{1}^{(3)}\left[\begin{array}{c}
a_{1}, a_{2}, c_{1}^{\prime \prime}, c_{2}^{\prime \prime} \\
\left.g_{1}^{\prime}, g_{2}^{\prime}, h_{1}, h_{2}, h_{3}, h_{1}^{\prime}, h_{1}^{\prime \prime} x, y, z\right]
\end{array}\right] \\
& =\sum_{i, j, k=0}^{\infty} \frac{\left(a_{1}\right)_{2 i+2 j+k}\left(a_{2}\right)_{2 i+2 j+k}\left(c_{1}^{\prime \prime}\right)_{k}\left(c_{2}^{\prime \prime}\right)_{k}}{\left(g_{1}^{\prime}\right)_{j+k}\left(g_{2}^{\prime}\right)_{j+k}\left(h_{1}\right)_{i}\left(h_{2}\right)_{i}\left(h_{3}\right)_{i}\left(h_{1}^{\prime}\right)_{j}\left(h_{1}^{\prime \prime}\right)_{k} i ! j ! k !} x^{i} y^{j} z^{k} ; \\
& \mathcal{G}_{2}^{(3)}\left[\begin{array}{c}
a_{1}, a_{2}, c_{1}^{\prime \prime}, c_{2}^{\prime \prime} \\
\left.h_{1}, h_{2}, h_{3}, h_{1}^{\prime}, h_{2}^{\prime}, h_{3}^{\prime}, h_{1}^{\prime \prime}, h_{2}^{\prime \prime}, h_{3}^{\prime \prime} x, y, z\right]
\end{array}\right. \\
& =\sum_{i, j, k=0}^{\infty} \frac{\left(a_{1}\right)_{2 i+2 j+k}\left(a_{2}\right)_{2 i+2 j+k}\left(c_{1}^{\prime \prime}\right)_{k}\left(c_{2}^{\prime \prime}\right)_{k}}{\left(h_{1}\right)_{i}\left(h_{2}\right)_{i}\left(h_{3}\right)_{i}\left(h_{1}^{\prime}\right)_{j}\left(h_{2}^{\prime}\right)_{j}\left(h_{3}^{\prime}\right)_{j}\left(h_{1}^{\prime \prime}\right)_{k}\left(h_{2}^{\prime \prime}\right)_{k}\left(h_{3}^{\prime \prime}\right)_{k} i ! j ! k !} x^{i} y^{j} z^{k} ;
\end{aligned}
$$


Several Integral Representations Involving Triple Hypergeometric Functions 131

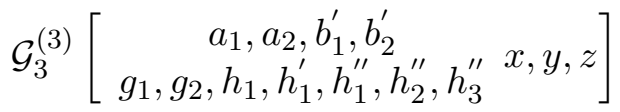

$$
\begin{aligned}
& =\sum_{i, j, k=0}^{\infty} \frac{\left(a_{1}\right)_{2 i+2 j+k}\left(a_{2}\right)_{2 i+2 j+k}\left(b_{1}^{\prime}\right)_{j+k}\left(b_{2}^{\prime}\right)_{j+k}}{\left(g_{1}\right)_{i+j}\left(g_{2}\right)_{i+j}\left(h_{1}\right)_{i}\left(h_{1}^{\prime}\right)_{j}\left(h_{1}^{\prime \prime}\right)_{k}\left(h_{2}^{\prime \prime}\right)_{k}\left(h_{3}^{\prime \prime}\right)_{k} i ! j ! k !} x^{i} y^{j} z^{k} ; \\
& \mathcal{G}_{4}^{(3)}\left[\begin{array}{c}
a_{1}, a_{2}, b_{1}^{\prime}, b_{2}^{\prime} \\
\left.h_{1}, h_{2}, h_{3}, h_{1}^{\prime}, h_{2}^{\prime}, h_{3}^{\prime}, h_{1}^{\prime \prime}, h_{2}^{\prime \prime}, h_{3}^{\prime \prime} x, y, z\right]
\end{array}\right. \\
& =\sum_{i, j, k=0}^{\infty} \frac{\left(a_{1}\right)_{2 i+2 j+k}\left(a_{2}\right)_{2 i+2 j+k}\left(b_{1}^{\prime}\right)_{j+k}\left(b_{2}^{\prime}\right)_{j+k}}{\left(h_{1}\right)_{i}\left(h_{2}\right)_{i}\left(h_{3}\right)_{i}\left(h_{1}^{\prime}\right)_{j}\left(h_{2}^{\prime}\right)_{j}\left(h_{3}^{\prime}\right)_{j}\left(h_{1}^{\prime \prime}\right)_{k}\left(h_{2}^{\prime \prime}\right)_{k}\left(h_{3}^{\prime \prime}\right)_{k} i ! j ! k !} x^{i} y^{j} z^{k} \text {; } \\
& \mathcal{G}_{5}^{(3)}\left[\begin{array}{c}
a_{1}, a_{2}, c_{1}^{\prime}, c_{2}^{\prime}, c_{1}^{\prime \prime}, c_{2}^{\prime \prime} \\
e_{1}, e_{2}, h_{1}, h_{1}^{\prime}, h_{1}^{\prime \prime}
\end{array} x, y, z\right] \\
& =\sum_{i, j, k=0}^{\infty} \frac{\left(a_{1}\right)_{2 i+2 j+k}\left(a_{2}\right)_{2 i+2 j+k}\left(c_{1}^{\prime}\right)_{j}\left(c_{2}^{\prime}\right)_{j}\left(c_{1}^{\prime \prime}\right)_{k}\left(c_{2}^{\prime \prime}\right)_{k}}{\left(e_{1}\right)_{i+j+k}\left(e_{2}\right)_{i+j+k}\left(h_{1}\right)_{i}\left(h_{1}^{\prime}\right)_{j}\left(h_{1}^{\prime \prime}\right)_{k} i ! j ! k !} x^{j} z^{k}
\end{aligned}
$$

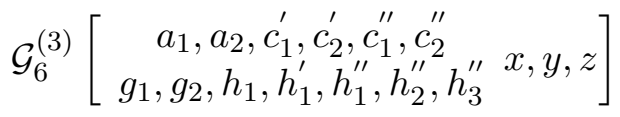

$$
\begin{aligned}
& =\sum_{i, j, k=0}^{\infty} \frac{\left(a_{1}\right)_{2 i+2 j+k}\left(a_{2}\right)_{2 i+2 j+k}\left(c_{1}^{\prime}\right)_{j}\left(c_{2}^{\prime}\right)_{j}\left(c_{1}^{\prime \prime}\right)_{k}\left(c_{2}^{\prime \prime}\right)_{k}}{\left(g_{1}\right)_{i+j}\left(g_{2}\right)_{i+j}\left(h_{1}\right)_{i}\left(h_{1}^{\prime}\right)_{j}\left(h_{1}^{\prime \prime}\right)_{k}\left(h_{2}^{\prime \prime}\right)_{k}\left(h_{3}^{\prime \prime}\right)_{k} i ! j ! k !} x^{i} y^{j} z^{k} ; \\
& \mathcal{G}_{7}^{(3)}\left[\begin{array}{c}
a_{1}, a_{2}, c_{1}^{\prime}, c_{2}^{\prime}, c_{1}^{\prime \prime}, c_{2}^{\prime \prime} \\
g_{1}^{\prime}, g_{2}^{\prime}, h_{1}, h_{2}, h_{3}, h_{1}^{\prime}, h_{2}^{\prime \prime} x, y, z
\end{array}\right] \\
& =\sum_{i, j, k=0}^{\infty} \frac{\left(a_{1}\right)_{2 i+2 j+k}\left(a_{2}\right)_{2 i+2 j+k}\left(c_{1}^{\prime}\right)_{j}\left(c_{2}^{\prime}\right)_{j}\left(c_{1}^{\prime \prime}\right)_{k}\left(c_{2}^{\prime \prime}\right)_{k}}{\left(g_{1}^{\prime}\right)_{j+k}\left(g_{2}^{\prime}\right)_{j+k}\left(h_{1}\right)_{i}\left(h_{2}\right)_{i}\left(h_{3}\right)_{i}\left(h_{1}^{\prime}\right)_{j}\left(h_{1}^{\prime \prime}\right)_{k} i ! j ! k !} x^{i} y^{j} z^{k} ; \\
& \mathcal{G}_{8}^{(3)}\left[\begin{array}{c}
a_{1}, a_{2}, c_{1}^{\prime}, c_{2}^{\prime}, c_{1}^{\prime \prime}, c_{2}^{\prime \prime} \\
h_{1}, h_{2}, h_{3}, h_{1}^{\prime}, h_{2}^{\prime}, h_{3}^{\prime}, h_{1}^{\prime \prime}, h_{2}^{\prime \prime}, h_{3}^{\prime \prime} x, y, z
\end{array}\right] \\
& =\sum_{i, j, k=0}^{\infty} \frac{\left(a_{1}\right)_{2 i+j+k}\left(a_{2}\right)_{2 i+j+k}\left(c_{1}^{\prime}\right)_{j}\left(c_{2}^{\prime}\right)_{j}\left(c_{1}^{\prime \prime}\right)_{k}\left(c_{2}^{\prime \prime}\right)_{k}}{\left(h_{1}\right)_{i}\left(h_{2}\right)_{i}\left(h_{3}\right)_{i}\left(h_{1}^{\prime}\right)_{j}\left(h_{2}^{\prime}\right)_{j}\left(h_{3}^{\prime}\right)_{j}\left(h_{1}^{\prime \prime}\right)_{k}\left(h_{2}^{\prime \prime}\right)_{k}\left(h_{3}^{\prime \prime}\right)_{k} i ! j ! k !} x^{i} y^{j} z^{k} ;
\end{aligned}
$$




$$
\begin{aligned}
& \mathcal{G}_{9}^{(3)}\left[\begin{array}{c}
a_{1}^{\prime}, a_{2}^{\prime}, a_{1}^{\prime \prime}, a_{2}^{\prime \prime} \\
\left.e_{1}, e_{2}, h_{1}, h_{1}^{\prime}, h_{1}^{\prime \prime} x, y, z\right]
\end{array}\right] \\
& =\sum_{i, j, k=0}^{\infty} \frac{\left(a_{1}^{\prime}\right)_{2 i+j}\left(a_{2}^{\prime}\right)_{2 i+j}\left(a_{1}^{\prime \prime}\right)_{j+2 k}\left(a_{2}^{\prime \prime}\right)_{j+2 k}}{\left(e_{1}\right)_{i+j+k}\left(e_{2}\right)_{i+j+k}\left(h_{1}\right)_{i}\left(h_{1}^{\prime}\right)_{j}\left(h_{1}^{\prime \prime}\right)_{k} i ! j ! k !} x^{i} y^{j} z^{k} ;
\end{aligned}
$$

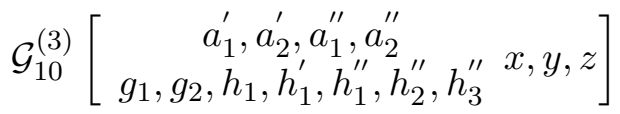

$$
\begin{aligned}
& =\sum_{i, j, k=0}^{\infty} \frac{\left(a_{1}^{\prime}\right)_{2 i+j}\left(a_{2}^{\prime}\right)_{2 i+j}\left(a_{1}^{\prime \prime}\right)_{j+2 k}\left(a_{2}^{\prime \prime}\right)_{j+2 k}}{\left(g_{1}\right)_{i+j}\left(g_{2}\right)_{i+j}\left(h_{1}\right)_{i}\left(h_{1}^{\prime}\right)_{j}\left(h_{1}^{\prime \prime}\right)_{k}\left(h_{2}^{\prime \prime}\right)_{k}\left(h_{3}^{\prime \prime}\right)_{k} i ! j ! k !} x^{i} y^{j} z^{k} ; \\
& \mathcal{G}_{11}^{(3)}\left[\begin{array}{c}
a_{1}^{\prime}, a_{2}^{\prime}, a_{1}^{\prime \prime}, a_{2}^{\prime \prime} \\
g_{1}^{\prime \prime}, g_{2}^{\prime \prime}, h_{1}, h_{1}^{\prime}, h_{2}^{\prime}, h_{3}^{\prime}, h_{1}^{\prime \prime} x, y, z
\end{array}\right] \\
& =\sum_{i, j, k=0}^{\infty} \frac{\left(a_{1}^{\prime}\right)_{2 i+j}\left(a_{2}^{\prime}\right)_{2 i+j}\left(a_{1}^{\prime \prime}\right)_{j+2 k}\left(a_{2}^{\prime \prime}\right)_{j+2 k}}{\left(g_{1}^{\prime \prime}\right)_{i+k}\left(g_{2}^{\prime \prime}\right)_{i+k}\left(h_{1}\right)_{i}\left(h_{1}^{\prime}\right)_{j}\left(h_{2}^{\prime}\right)_{j}\left(h_{3}^{\prime}\right)_{j}\left(h_{1}^{\prime \prime}\right)_{k} i ! j ! k !} x^{i} y^{j} z^{k} ; \\
& \mathcal{G}_{12}^{(3)}\left[\begin{array}{c}
a_{1}^{\prime}, a_{2}^{\prime}, a_{1}^{\prime \prime}, a_{2}^{\prime \prime} \\
\left.h_{1}, h_{2}, h_{3}, h_{1}^{\prime}, h_{2}^{\prime}, h_{3}^{\prime}, h_{1}^{\prime \prime}, h_{2}^{\prime \prime}, h_{3}^{\prime \prime} x, y, z\right]
\end{array}\right] \\
& =\sum_{i, j, k=0}^{\infty} \frac{\left(a_{1}^{\prime}\right)_{2 i+j}\left(a_{2}^{\prime}\right)_{2 i+j}\left(a_{1}^{\prime \prime}\right)_{j+2 k}\left(a_{2}^{\prime \prime}\right)_{j+2 k}}{\left(h_{1}\right)_{i}\left(h_{2}\right)_{i}\left(h_{3}\right)_{i}\left(h_{1}^{\prime}\right)_{j}\left(h_{2}^{\prime}\right)_{j}\left(h_{3}^{\prime}\right)_{j}\left(h_{1}^{\prime \prime}\right)_{k}\left(h_{2}^{\prime \prime}\right)_{k}\left(h_{3}^{\prime \prime}\right)_{k} i ! j ! k !} x^{i} y^{j} z^{k} \text {; } \\
& \mathcal{G}_{13}^{(3)}\left[\begin{array}{c}
a_{1}^{\prime}, a_{2}^{\prime}, b_{1}^{\prime}, b_{2}^{\prime}, c_{1}^{\prime \prime}, c_{2}^{\prime \prime} \\
e_{1}, e_{2}, h_{1}, h_{1}^{\prime}, h_{1}^{\prime \prime}
\end{array} x, y, z\right] \\
& =\sum_{i, j, k=0}^{\infty} \frac{\left(a_{1}^{\prime}\right)_{2 i+j}\left(a_{2}^{\prime}\right)_{2 i+j}\left(b_{1}^{\prime}\right)_{j+k}\left(b_{2}^{\prime}\right)_{j+k}\left(c_{1}^{\prime \prime}\right)_{k}\left(c_{2}^{\prime \prime}\right)_{k}}{\left(e_{1}\right)_{i+j+k}\left(e_{2}\right)_{i+j+k}\left(h_{1}\right)_{i}\left(h_{1}^{\prime}\right)_{j}\left(h_{1}^{\prime \prime}\right)_{k} i ! j ! k !} x^{j} y^{j} z^{k} \\
& \mathcal{G}_{14}^{(3)}\left[\begin{array}{c}
a_{1}^{\prime}, a_{2}^{\prime}, b_{1}^{\prime}, b_{2}^{\prime}, c_{1}^{\prime \prime}, c_{2}^{\prime \prime} \\
\left.g_{1}, g_{2}, h_{1}, h_{1}^{\prime}, h_{1}^{\prime \prime}, h_{2}^{\prime \prime}, h_{3}^{\prime \prime} x, y, z\right]
\end{array}\right] \\
& =\sum_{i, j, k=0}^{\infty} \frac{\left(a_{1}^{\prime}\right)_{2 i+j}\left(a_{2}^{\prime}\right)_{2 i+j}\left(b_{1}^{\prime}\right)_{j+k}\left(b_{2}^{\prime}\right)_{j+k}\left(c_{1}^{\prime \prime}\right)_{k}\left(c_{2}^{\prime \prime}\right)_{k}}{\left(g_{1}\right)_{i+j}\left(g_{2}\right)_{i+j}\left(h_{1}\right)_{i}\left(h_{1}^{\prime}\right)_{j}\left(h_{1}^{\prime \prime}\right)_{k}\left(h_{2}^{\prime \prime}\right)_{k}\left(h_{3}^{\prime \prime}\right)_{k} i ! j ! k !} x^{i} y^{j} z^{k} ;
\end{aligned}
$$


Several Integral Representations Involving Triple Hypergeometric Functions 133

$$
\begin{aligned}
& \mathcal{G}_{15}^{(3)}\left[\begin{array}{c}
a_{1}^{\prime}, a_{2}^{\prime}, b_{1}^{\prime}, b_{2}^{\prime}, c_{1}^{\prime \prime}, c_{2}^{\prime \prime} \\
g_{1}^{\prime}, g_{2}^{\prime}, h_{1}, h_{2}, h_{3}, h_{1}^{\prime}, h_{1}^{\prime \prime} x, y, z
\end{array}\right] \\
& =\sum_{i, j, k=0}^{\infty} \frac{\left(a_{1}^{\prime}\right)_{2 i+j}\left(a_{2}^{\prime}\right)_{2 i+j}\left(b_{1}^{\prime}\right)_{j+k}\left(b_{2}^{\prime}\right)_{j+k}\left(c_{1}^{\prime \prime}\right)_{k}\left(c_{2}^{\prime \prime}\right)_{k}}{\left(g_{1}^{\prime}\right)_{j+k}\left(g_{2}^{\prime}\right)_{j+k}\left(h_{1}\right)_{i}\left(h_{2}\right)_{i}\left(h_{3}\right)_{i}\left(h_{1}^{\prime}\right)_{j}\left(h_{1}^{\prime \prime}\right)_{k} i ! j ! k !} x^{i} y^{j} z^{k} ;
\end{aligned}
$$

$$
\begin{aligned}
& \mathcal{G}_{16}^{(3)}\left[\begin{array}{c}
a_{1}^{\prime}, a_{2}^{\prime}, b_{1}^{\prime}, b_{2}^{\prime}, c_{1}^{\prime \prime}, c_{2}^{\prime \prime} \\
\left.g_{1}^{\prime \prime}, g_{2}^{\prime \prime}, h_{1}, h_{1}^{\prime}, h_{2}^{\prime}, h_{3}^{\prime}, h_{1}^{\prime \prime} x, y, z\right]
\end{array}\right] \\
& =\sum_{i, j, k=0}^{\infty} \frac{\left(a_{1}^{\prime}\right)_{2 i+j}\left(a_{2}^{\prime}\right)_{2 i+j}\left(b_{1}^{\prime}\right)_{j+k}\left(b_{2}^{\prime}\right)_{j+k}\left(c_{1}^{\prime \prime}\right)_{k}\left(c_{2}^{\prime \prime}\right)_{k}}{\left(g_{1}^{\prime \prime}\right)_{i+k}\left(g_{2}^{\prime \prime}\right)_{i+k}\left(h_{1}\right)_{i}\left(h_{1}^{\prime}\right)_{j}\left(h_{2}^{\prime}\right)_{j}\left(h_{3}^{\prime}\right)_{j}\left(h_{1}^{\prime \prime}\right)_{k} i ! j ! k !} x^{i} y^{j} z^{k} ;
\end{aligned}
$$

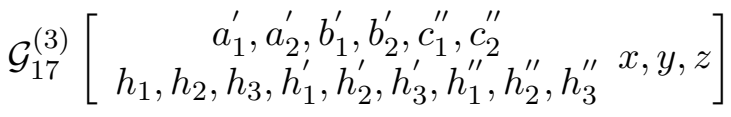

$$
\begin{aligned}
& =\sum_{i, j, k=0}^{\infty} \frac{\left(a_{1}^{\prime}\right)_{2 i+j}\left(a_{2}^{\prime}\right)_{2 i+j}\left(b_{1}^{\prime}\right)_{j+k}\left(b_{2}^{\prime}\right)_{j+k}\left(c_{1}^{\prime \prime}\right)_{k}\left(c_{2}^{\prime \prime}\right)_{k}}{\left(h_{1}\right)_{i}\left(h_{2}\right)_{i}\left(h_{3}\right)_{i}\left(h_{1}^{\prime}\right)_{j}\left(h_{2}^{\prime}\right)_{j}\left(h_{3}^{\prime}\right)_{j}\left(h_{1}^{\prime \prime}\right)_{k}\left(h_{2}^{\prime \prime}\right)_{k}\left(h_{3}^{\prime \prime}\right)_{k} i ! j ! k !} x^{i} y^{j} z^{k} ;
\end{aligned}
$$

$$
\begin{aligned}
\mathcal{G}_{18}^{(3)} & \left.\begin{array}{c}
a_{1}^{\prime}, a_{2}^{\prime}, c_{1}^{\prime}, c_{2}^{\prime}, c_{1}^{\prime \prime}, c_{2}^{\prime \prime}, c_{3}^{\prime \prime}, c_{4}^{\prime \prime} \\
e_{1}, e_{2}, h_{1}, h_{1}^{\prime}, h_{1}^{\prime \prime}
\end{array} \quad x, y, z\right] \\
= & \sum_{i, j, k=0}^{\infty} \frac{\left(a_{1}^{\prime}\right)_{2 i+j}\left(a_{2}^{\prime}\right)_{2 i+j}\left(c_{1}^{\prime}\right)_{j}\left(c_{2}^{\prime}\right)_{j}\left(c_{1}^{\prime \prime}\right)_{k}\left(c_{2}^{\prime \prime}\right)_{k}\left(c_{3}^{\prime \prime}\right)_{k}\left(c_{4}^{\prime \prime}\right)_{k}}{\left(e_{1}\right)_{i+j+k}\left(e_{2}\right)_{i+j+k}\left(h_{1}\right)_{i}\left(h_{1}^{\prime}\right)_{j}\left(h_{1}^{\prime \prime}\right)_{k} i ! j ! k !} x^{j} y^{j} z^{k} ;
\end{aligned}
$$

$$
\begin{aligned}
\mathcal{G}_{19}^{(3)} & {\left[\begin{array}{c}
a_{1}^{\prime}, a_{2}^{\prime}, c_{1}^{\prime}, c_{2}^{\prime}, c_{1}^{\prime \prime}, c_{2}^{\prime \prime}, c_{3}^{\prime \prime}, c_{4}^{\prime \prime} \\
g_{1}^{\prime}, g_{2}, h_{1}, h_{2}, h_{3}, h_{1}^{\prime}, h_{1}^{\prime \prime}
\end{array} x, y, z\right] } \\
= & \sum_{i, j, k=0}^{\infty} \frac{\left(a_{1}^{\prime}\right)_{2 i+j}\left(a_{2}^{\prime}\right)_{2 i+j}\left(c_{1}^{\prime}\right)_{j}\left(c_{2}^{\prime}\right)_{j}\left(c_{1}^{\prime \prime}\right)_{k}\left(c_{2}^{\prime \prime}\right)_{k}\left(c_{3}^{\prime \prime}\right)_{k}\left(c_{4}^{\prime \prime}\right)_{k}}{\left(g_{1}^{\prime}\right)_{j+k}\left(g_{2}^{\prime}\right)_{j+k}\left(h_{1}\right)_{i}\left(h_{2}\right)_{i}\left(h_{3}\right)_{i}\left(h_{1}^{\prime}\right)_{j}\left(h_{1}^{\prime \prime}\right)_{k} i ! j ! k !} x^{i} y^{j} z^{k}
\end{aligned}
$$




$$
\begin{aligned}
& \mathcal{G}_{20}^{(3)}\left[\begin{array}{c}
a_{1}^{\prime}, a_{2}^{\prime}, c_{1}^{\prime}, c_{2}^{\prime}, c_{1}^{\prime \prime}, c_{2}^{\prime \prime}, c_{3}^{\prime \prime}, c_{4}^{\prime \prime} \\
\left.g_{1}^{\prime \prime}, g_{2}^{\prime \prime}, h_{1}, h_{1}^{\prime}, h_{2}^{\prime}, h_{3}^{\prime}, h_{1}^{\prime \prime} \quad x, y, z\right]
\end{array}\right]
\end{aligned}
$$

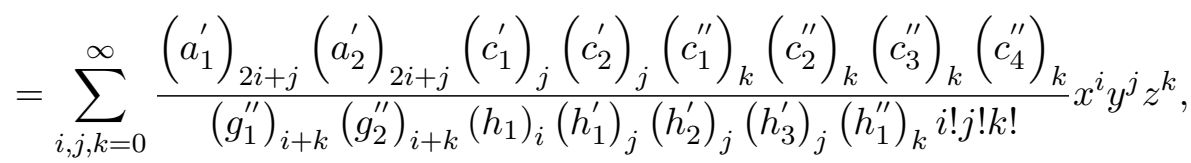

where $(\lambda)_{n}$ is the Pochhammer symbol defined (for $\lambda \in \mathbb{C}$ ) by (see $[9]$ ):

$$
\begin{aligned}
& (\lambda)_{n}:=\left\{\begin{array}{cl}
1 & (n=0) \\
\lambda(\lambda+1) \ldots \lambda(\lambda+n-1) & (n \in \mathbb{N}:=\{1,2,3, \ldots\})
\end{array}\right. \\
& =\frac{\Gamma(\lambda+n)}{\Gamma(\lambda)} \quad\left(\lambda \in \mathbb{C} \backslash \mathbb{Z}_{0}^{-}\right),
\end{aligned}
$$

$\Gamma(z)$ being the well-known Gamma function, $\mathbb{C}$ and $\mathbb{Z}_{0}^{-}$denoting the set of complex numbers and the set of nonpositive integers, respectively.

\section{Integral Representations of Laplace Type}

In this section by employing the Laplace integral representation formula $[2]$

$$
(a)_{m}=\frac{1}{\Gamma(a)} \int_{0}^{\infty} e^{-t} t^{a+m-1} d t \quad\left(\Re(a)>0 ; m \in \mathbb{N}_{0}:=\mathbb{N} \cup\{0\}\right)
$$

to the series representations of aforementioned functions $\mathcal{G}_{1}^{(3)}, \ldots, \mathcal{G}_{20}^{(3)}$, we present certain integral representations of Laplace type. The regions of convergence of the series (1.2) to (1.21) may be investigated by means of Horn's theorem for triple series (see [10, Chapter 5, pp. 128-134]). For instance, the region of convergence for hypergeometric function $\mathcal{G}_{2}^{(3)}$ is given as follows:

$$
\left\{(x, y, z):|x|<\frac{1}{16},|y|<\frac{1}{16},|z|<1, r:=|x|, s:=|y|, t:=|z|, \sqrt[4]{r}+\sqrt[4]{s}+\sqrt{t}=1\right\} .
$$


Several Integral Representations Involving Triple Hypergeometric Functions 135

By means of formula (2.1) we find the following integral representations of Laplace type:

$(2.2)$

$$
\begin{aligned}
& \mathcal{G}_{1}^{(3)}\left[\begin{array}{c}
a_{1}, a_{2}, c_{1}^{\prime \prime}, c_{2}^{\prime \prime} \\
\left.g_{1}^{\prime}, g_{2}^{\prime}, h_{1}, h_{2}, h_{3}, h_{1}^{\prime}, h_{1}^{\prime \prime} x, y, z\right]
\end{array}\right. \\
& =\frac{1}{\Gamma\left(a_{1}\right) \Gamma\left(a_{2}\right)} \int_{0}^{\infty} \int_{0}^{\infty} e^{-\left(\xi_{1}+\xi_{2}\right)} \xi_{1}^{a_{1}-1} \xi_{2}^{a_{2}-1}{ }_{0} F_{3}\left(h_{1}, h_{2}, h_{3} ; x \xi_{1}^{2} \xi_{2}^{2}\right) \\
& \cdot F_{2: 1 ; 1}^{0: 0 ; 2}\left[\begin{array}{rrr}
-: & -; & c_{1}^{\prime \prime}, c_{2}^{\prime \prime} ; \\
g_{1}^{\prime}, g_{2}^{\prime}: & h_{1}^{\prime} ; & h_{1}^{\prime \prime} ;
\end{array} \xi_{1}^{2} \xi_{2}^{2}, z \xi_{1} \xi_{2}\right] d \xi_{1} d \xi_{2} \\
& \left(\Re\left(a_{1}\right)>0, \Re\left(a_{2}\right)>0\right) \text {; }
\end{aligned}
$$

$$
\begin{aligned}
\mathcal{G}_{2}^{(3)} & {\left[\begin{array}{c}
a_{1}, a_{2}, c_{1}^{\prime \prime}, c_{2}^{\prime \prime} \\
\left.h_{1}, h_{2}, h_{3}, h_{1}^{\prime}, h_{2}^{\prime}, h_{3}^{\prime}, h_{1}^{\prime \prime}, h_{2}^{\prime \prime}, h_{3}^{\prime \prime} x, y, z\right]
\end{array}\right.} \\
= & \frac{1}{\Gamma\left(a_{1}\right) \Gamma\left(a_{2}\right)} \int_{0}^{\infty} \int_{0}^{\infty} e^{-\left(\xi_{1}+\xi_{2}\right)} \xi_{1}^{a_{1}-1} \xi_{2}^{a_{2}-1} \cdot{ }_{0} F_{3}\left(h_{1}, h_{2}, h_{3} ; x \xi_{1}^{2} \xi_{2}^{2}\right) \\
& { }_{0} F_{3}\left(h_{1}^{\prime}, h_{2}^{\prime}, h_{3}^{\prime} ; y \xi_{1}^{2} \xi_{2}^{2}\right){ }_{2} F_{3}\left(c_{1}^{\prime \prime}, c_{2}^{\prime \prime} ; h_{1}^{\prime \prime}, h_{2}^{\prime \prime}, h_{3}^{\prime \prime} ; z \xi_{1} \xi_{2}\right) \\
& d \xi_{1} d \xi_{2}\left(\Re\left(a_{1}\right)>0, \Re\left(a_{2}\right)>0\right) ;
\end{aligned}
$$

$$
\begin{aligned}
& \mathcal{G}_{3}^{(3)}\left[\begin{array}{c}
a_{1}, a_{2}, b_{1}^{\prime}, b_{2}^{\prime} \\
\left.g_{1}, g_{2}, h_{1}, h_{1}^{\prime}, h_{1}^{\prime \prime}, h_{2}^{\prime \prime}, h_{3}^{\prime \prime} x, y, z\right]
\end{array}\right] \\
& =\frac{1}{\Gamma\left(a_{1}\right) \Gamma\left(a_{2}\right) \Gamma\left(b_{1}^{\prime}\right) \Gamma\left(b_{2}^{\prime}\right)} \int_{0}^{\infty} \cdots \int_{0}^{\infty} e^{-\left(\xi_{1}+\xi_{2}+\xi_{3}+\xi_{4}\right)} \\
& \cdot \xi_{1}^{a_{1}-1} \xi_{2}^{a_{2}-1} \xi_{3}^{b_{1}^{\prime}-1} \xi_{4}^{b_{2}^{\prime}-1} F_{2: 1 ; 1}^{0: 0 ; 0}\left[\begin{array}{rrr}
-: & -; & -; \\
g_{1}, g_{2}: & h_{1} ; & h_{1}^{\prime} ;
\end{array} \xi_{1}^{2} \xi_{2}^{2}, y \xi_{1}^{2} \xi_{2}^{2} \xi_{3} \xi_{4}\right] \\
& \cdot{ }_{0} F_{3}\left(h_{1}^{\prime \prime}, h_{2}^{\prime \prime}, h_{3}^{\prime \prime} ; z \xi_{1} \xi_{2} \xi_{3} \xi_{4}\right) d \xi_{1} d \xi_{2} d \xi_{3} d \xi_{4} \\
& \left(\Re\left(a_{1}\right)>0, \Re\left(a_{2}\right)>0, \Re\left(b_{1}^{\prime}\right)>0, \Re\left(b_{2}^{\prime}\right)>0\right) ;
\end{aligned}
$$


$(2.5)$

$$
\begin{aligned}
\mathcal{G}_{4}^{(3)}\left[\begin{array}{c}
a_{1}, a_{2}, b_{1}^{\prime}, b_{2}^{\prime} \\
\left.h_{1}, h_{2}, h_{3}, h_{1}^{\prime}, h_{2}^{\prime}, h_{3}^{\prime}, h_{1}^{\prime \prime}, h_{2}^{\prime \prime}, h_{3}^{\prime \prime} x, y, z\right]
\end{array}\right. \\
\quad=\frac{1}{\Gamma\left(a_{1}\right) \Gamma\left(a_{2}\right)} \int_{0}^{\infty} \int_{0}^{\infty} e^{-\left(\xi_{1}+\xi_{2}\right)} \xi_{1}^{a_{1}-1} \xi_{2}^{a_{2}-1} \cdot{ }_{0} F_{3}\left(h_{1}, h_{2}, h_{1} ; x \xi_{1}^{2} \xi_{2}^{2}\right) \\
\quad F_{0: 3 ; 3}^{2: 0 ; 0}\left[\begin{array}{c}
b_{1}^{\prime}, b_{2}^{\prime}: \\
-:
\end{array} \quad h_{1}^{\prime}, h_{2}^{\prime}, h_{3}^{\prime} ; \quad h_{1}^{\prime \prime}, h_{2}^{\prime \prime}, h_{3}^{\prime \prime} ; y \xi_{1}^{2} \xi_{2}^{2}, z \xi_{1} \xi_{2}\right] d \xi_{1} d \xi_{2} \\
\quad\left(\Re\left(a_{1}\right)>0, \Re\left(a_{2}\right)>0\right) ;
\end{aligned}
$$

$$
\begin{aligned}
\mathcal{G}_{5}^{(3)} & {\left[\begin{array}{c}
a_{1}, a_{2}, c_{1}^{\prime}, c_{2}^{\prime}, c_{1}^{\prime \prime}, c_{2}^{\prime \prime} \\
e_{1}, e_{2}, h_{1}, h_{1}^{\prime}, h_{1}^{\prime \prime}
\end{array} x, y, z\right] } \\
& =\frac{1}{\Gamma\left(a_{1}\right) \Gamma\left(a_{2}\right)} \int_{0}^{\infty} \int_{0}^{\infty} e^{-\left(\xi_{1}+\xi_{2}\right)} \xi_{1}^{a_{1}-1} \xi_{2}^{a_{2}-1} \cdot F^{(3)} \\
& {\left[\begin{array}{c}
c_{1}^{\prime}, c_{2}^{\prime}, c_{1}^{\prime \prime}, c_{2}^{\prime \prime} \\
e_{1}, e_{2}, h_{1}, h_{1}^{\prime}, h_{1}^{\prime \prime}
\end{array} x \xi_{1}^{2} \xi_{2}^{2}, y \xi_{1}^{2} \xi_{2}^{2}, z \xi_{1} \xi_{2}\right] d \xi_{1} d \xi_{2}\left(\Re\left(a_{1}\right)>0, \Re\left(a_{2}\right)>0\right), }
\end{aligned}
$$

where $F^{(3)}$ is the generalized Srivastava's hypergeometric function:

$$
\begin{aligned}
& F^{(3)}\left[\begin{array}{c}
c_{1}^{\prime}, c_{2}^{\prime}, c_{1}^{\prime \prime}, c_{2}^{\prime \prime} \\
\left.e_{1}, e_{2}, h_{1}, h_{1}^{\prime}, h_{1}^{\prime \prime} x, y, z\right]
\end{array}\right] \\
& =\sum_{i, j, k=0}^{\infty} \frac{\left(c_{1}^{\prime}\right)_{j}\left(c_{2}^{\prime}\right)_{j}\left(c_{1}^{\prime \prime}\right)_{k}\left(c_{2}^{\prime \prime}\right)_{k}}{\left(e_{1}\right)_{i+j+k}\left(e_{2}\right)_{i+j+k}\left(h_{1}\right)_{i}\left(h_{1}^{\prime}\right)_{j}\left(h_{1}^{\prime \prime}\right)_{k} i ! j ! k !} x^{i} y^{j} z^{k} ;
\end{aligned}
$$

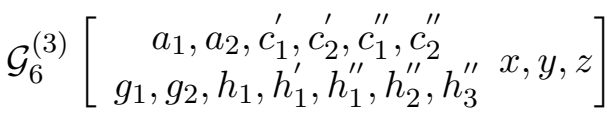

$$
\begin{aligned}
& =\frac{1}{\Gamma\left(a_{1}\right) \Gamma\left(a_{2}\right)} \int_{0}^{\infty} \int_{0}^{\infty} e^{-\left(\xi_{1}+\xi_{2}\right)} \xi_{1}^{a_{1}-1} \xi_{2}^{a_{2}-1}
\end{aligned}
$$

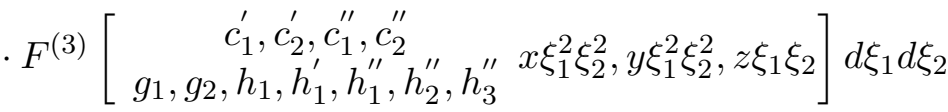

$$
\begin{aligned}
& \left(\Re\left(a_{1}\right)>0, \Re\left(a_{2}\right)>0\right) \text {, }
\end{aligned}
$$


where

$$
\begin{aligned}
& F^{(3)}\left[\begin{array}{c}
c_{1}^{\prime}, c_{2}^{\prime}, c_{1}^{\prime \prime}, c_{2}^{\prime \prime} \\
\left.g_{1}, g_{2}, h_{1}, h_{1}^{\prime}, h_{1}^{\prime \prime}, h_{2}^{\prime \prime}, h_{3}^{\prime \prime} x, y, z\right]
\end{array}\right. \\
& =\sum_{i, j, k=0}^{\infty} \frac{\left(c_{1}^{\prime}\right)_{j}\left(c_{2}^{\prime}\right)_{j}\left(c_{1}^{\prime \prime}\right)_{k}\left(c_{2}^{\prime \prime}\right)_{k}}{\left(g_{1}\right)_{i+j}\left(g_{2}\right)_{i+j}\left(h_{1}\right)_{i}\left(h_{1}^{\prime}\right)_{j}\left(h_{1}^{\prime \prime}\right)_{k}\left(h_{2}^{\prime \prime}\right)_{k}\left(h_{3}^{\prime \prime}\right)_{k} i ! j ! k !} x^{i} y^{j} z^{k} ;
\end{aligned}
$$

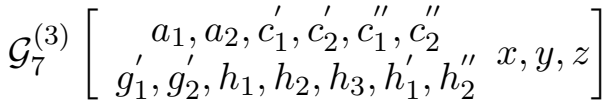

$$
\begin{aligned}
& =\frac{1}{\Gamma\left(a_{1}\right) \Gamma\left(a_{2}\right)} \int_{0}^{\infty} \int_{0}^{\infty} e^{-\left(\xi_{1}+\xi_{2}\right)} \xi_{1}^{a_{1}-1} \xi_{2}^{a_{2}-1}
\end{aligned}
$$

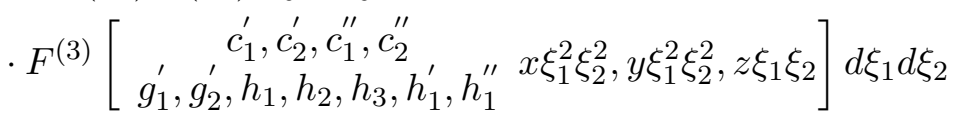

$$
\begin{aligned}
& \left(\Re\left(a_{1}\right)>0, \Re\left(a_{2}\right)>0\right) \text {, }
\end{aligned}
$$

where

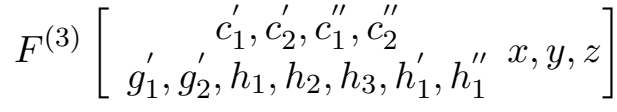

$$
\begin{aligned}
& =\sum_{i, j, k=0}^{\infty} \frac{\left(c_{1}^{\prime}\right)_{j}\left(c_{2}^{\prime}\right)_{j}\left(c_{1}^{\prime \prime}\right)_{k}\left(c_{2}^{\prime \prime}\right)_{k}}{\left(g_{1}^{\prime}\right)_{j+k}\left(g_{2}^{\prime}\right)_{j+k}\left(h_{1}\right)_{i}\left(h_{2}\right)_{i}\left(h_{3}\right)_{i}\left(h_{1}^{\prime}\right)_{j}\left(h_{1}^{\prime \prime}\right)_{k} i ! j ! k !} x^{i} y^{j} z^{k} \text {; }
\end{aligned}
$$

$$
\begin{gathered}
\mathcal{G}_{8}^{(3)}\left[\begin{array}{c}
a_{1}, a_{2}, c_{1}^{\prime}, c_{2}^{\prime}, c_{1}^{\prime \prime}, c_{2}^{\prime \prime} \\
\left.h_{1}, h_{2}, h_{3}, h_{1}^{\prime}, h_{2}^{\prime}, h_{3}^{\prime}, h_{1}^{\prime \prime}, h_{2}^{\prime \prime}, h_{3}^{\prime \prime} x, y, z\right]
\end{array}\right. \\
=\frac{1}{\Gamma\left(a_{1}\right) \Gamma\left(a_{2}\right)} \int_{0}^{\infty} \int_{0}^{\infty} e^{-\left(\xi_{1}+\xi_{2}\right)} \xi_{1}^{a_{1}-1} \xi_{2}^{a_{2}-1}{ }_{0} F_{3}\left(h_{1}, h_{2}, h_{3} ; x \xi_{1}^{2} \xi_{2}^{2}\right) \\
\cdot{ }_{2} F_{3}\left(c_{1}^{\prime}, c_{2}^{\prime} ; h_{1}^{\prime}, h_{2}^{\prime}, h_{3}^{\prime} ; y \xi_{1}^{2} \xi_{2}^{2}\right){ }_{2} F_{3}\left(c_{1}^{\prime \prime}, c_{2}^{\prime \prime} ; h_{1}^{\prime \prime}, h_{2}^{\prime \prime}, h_{3}^{\prime \prime} ; z \xi_{1} \xi_{2}\right) d \xi_{1} d \xi_{2} \\
\left(\Re\left(a_{1}\right)>0, \Re\left(a_{2}\right)>0\right) ;
\end{gathered}
$$


(2.13)

$$
\begin{aligned}
& \mathcal{G}_{9}^{(3)}\left[\begin{array}{c}
a_{1}^{\prime}, a_{2}^{\prime}, a_{1}^{\prime \prime}, a_{2}^{\prime \prime} \\
\left.e_{1}, e_{2}, h_{1}, h_{1}^{\prime}, h_{1}^{\prime \prime} x, y, z\right]
\end{array}\right. \\
& =\frac{1}{\Gamma\left(a_{1}^{\prime}\right) \Gamma\left(a_{2}^{\prime}\right) \Gamma\left(a_{1}^{\prime \prime}\right) \Gamma\left(a_{2}^{\prime \prime}\right)} \int_{0}^{\infty} \cdots \int_{0}^{\infty} e^{-\left(\xi_{1}+\xi_{2}+\xi_{3}+\xi_{4}\right)} \\
& \xi_{1}^{a_{1}^{\prime}-1} \xi_{2}^{a_{2}^{\prime}-1} \xi_{3}^{a_{1}^{\prime \prime}-1} \xi_{4}^{a_{2}^{\prime \prime}-1} \cdot F^{(3)}\left[e_{1}, e_{2}, h_{1}, h_{1}^{\prime}, h_{1}^{\prime \prime} x \xi_{1}^{2} \xi_{2}^{2}, y \xi_{1} \xi_{2} \xi_{3} \xi_{4}, z \xi_{3}^{2} \xi_{4}^{2}\right] \\
& d \xi_{1} \cdots d \xi_{4}\left(\Re\left(a_{1}^{\prime}\right)>0, \Re\left(a_{2}^{\prime}\right)>0, \Re\left(a_{1}^{\prime \prime}\right)>0, \Re\left(a_{2}^{\prime \prime}\right)>0\right),
\end{aligned}
$$

where

$$
\begin{aligned}
& F^{(3)}\left[e_{1}, e_{2}, h_{1}, h_{1}^{\prime}, h_{1}^{\prime \prime} x, y, z\right] \\
& =\sum_{i, j, k=0}^{\infty} \frac{1}{\left(e_{1}\right)_{i+j+k}\left(e_{2}\right)_{i+j+k}\left(h_{1}\right)_{i}\left(h_{1}^{\prime}\right)_{j}\left(h_{1}^{\prime \prime}\right)_{k} i ! j ! k !} x^{i} y^{j} z^{k}
\end{aligned}
$$

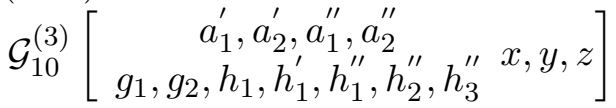

$$
\begin{aligned}
& =\frac{1}{\Gamma\left(a_{1}^{\prime}\right) \Gamma\left(a_{2}^{\prime}\right) \Gamma\left(a_{1}^{\prime \prime}\right) \Gamma\left(a_{2}^{\prime \prime}\right)} \int_{0}^{\infty} \cdots \int_{0}^{\infty} e^{-\left(\xi_{1}+\xi_{2}+\xi_{3}+\xi_{4}\right)} \\
& \xi_{1}^{a_{1}^{\prime}-1} \xi_{2}^{a_{2}^{\prime}-1} \xi_{3}^{a_{1}^{\prime \prime}-1} \xi_{4}^{a_{2}^{\prime \prime}-1} \cdot F_{2: 1 ; 1}^{0: 0 ; 0}\left[\begin{array}{rrr}
-: & -; & -; \\
g_{1}, g_{2}: & h_{1} ; & h_{1}^{\prime} ;
\end{array} \xi_{1}^{2} \xi_{2}^{2}, y \xi_{1} \xi_{2} \xi_{3} \xi_{4}\right] \\
& { }_{0} F_{3}\left(h_{1}^{\prime \prime}, h_{2}^{\prime \prime}, h_{3}^{\prime \prime} ; z \xi_{3}^{2} \xi_{4}^{2}\right) \\
& d \xi_{1} \cdots d \xi_{4}\left(\Re\left(a_{1}^{\prime}\right)>0, \Re\left(a_{2}^{\prime}\right)>0, \Re\left(a_{1}^{\prime \prime}\right)>0, \Re\left(a_{2}^{\prime \prime}\right)>0\right) ;
\end{aligned}
$$

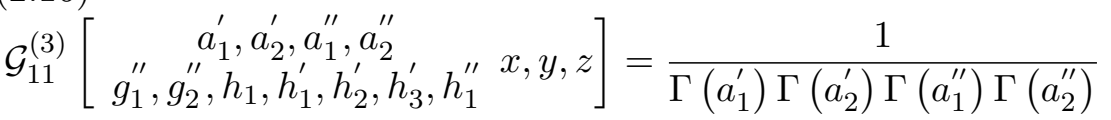

$$
\begin{aligned}
& \cdot \int_{0}^{\infty} \cdots \int_{0}^{\infty} e^{-\left(\xi_{1}+\xi_{2}+\xi_{3}+\xi_{4}\right)} \xi_{1}^{a_{1}^{\prime}-1} \xi_{2}^{a_{2}^{\prime}-1} \xi_{3}^{a_{1}^{\prime \prime}-1} \xi_{4}^{a_{2}^{\prime \prime}-1} \\
& \cdot F_{2: 1 ; 1}^{0: 0 ; 0}\left[\begin{array}{rrr}
-: & -; & -; \\
g_{1}^{\prime \prime}, g_{2}^{\prime \prime}: & h_{1} ; & h_{1}^{\prime \prime} ;
\end{array} \xi_{1}^{2} \xi_{2}^{2}, z \xi_{3}^{2} \xi_{4}^{2}\right]{ }_{0} F_{3}\left(h_{1}^{\prime}, h_{2}^{\prime}, h_{3}^{\prime} ; y \xi_{1} \xi_{2} \xi_{3} \xi_{4}\right) \\
& d \xi_{1} \cdots d \xi_{4}\left(\Re\left(a_{1}^{\prime}\right)>0, \Re\left(a_{2}^{\prime}\right)>0, \Re\left(a_{1}^{\prime \prime}\right)>0, \Re\left(a_{2}^{\prime \prime}\right)>0\right) ;
\end{aligned}
$$


Several Integral Representations Involving Triple Hypergeometric Functions 139

(2.17)

$$
\begin{gathered}
\mathcal{G}_{12}^{(3)}\left[\begin{array}{c}
a_{1}^{\prime}, a_{2}^{\prime}, a_{1}^{\prime \prime}, a_{2}^{\prime \prime} \\
\left.h_{1}, h_{2}, h_{3}, h_{1}^{\prime}, h_{2}^{\prime}, h_{3}^{\prime}, h_{1}^{\prime \prime}, h_{2}^{\prime \prime}, h_{3}^{\prime \prime} x, y, z\right]
\end{array}\right. \\
=\frac{1}{\Gamma\left(a_{1}^{\prime}\right) \Gamma\left(a_{2}^{\prime}\right) \Gamma\left(a_{1}^{\prime \prime}\right) \Gamma\left(a_{2}^{\prime \prime}\right)} \int_{0}^{\infty} \cdots \int_{0}^{\infty} e^{-\left(\xi_{1}+\xi_{2}+\xi_{3}+\xi_{4}\right)} \\
\xi_{1}^{a_{1}^{\prime}-1} \xi_{2}^{a_{2}^{\prime}-1} \xi_{3}^{\prime \prime}-1 \xi_{4}^{a_{2}^{\prime \prime}-1} \cdot{ }_{0} F_{3}\left(h_{1}, h_{2}, h_{3} ; x \xi_{1}^{2} \xi_{2}^{2}\right) \\
{ }_{0} F_{3}\left(h_{1}^{\prime}, h_{2}^{\prime}, h_{3}^{\prime} ; y \xi_{1} \xi_{2} \xi_{3} \xi_{4}\right){ }_{0} F_{3}\left(h_{1}^{\prime \prime}, h_{2}^{\prime \prime}, h_{3}^{\prime \prime} ; z \xi_{3}^{2} \xi_{4}^{2}\right) d \xi_{1} \cdots d \xi_{4} \\
\left(\Re\left(a_{1}^{\prime}\right)>0, \Re\left(a_{2}^{\prime}\right)>0, \Re\left(a_{1}^{\prime \prime}\right)>0, \Re\left(a_{2}^{\prime \prime}\right)>0\right) ;
\end{gathered}
$$

$$
\begin{aligned}
& \mathcal{G}_{13}^{(3)}\left[\begin{array}{c}
a_{1}^{\prime}, a_{2}^{\prime}, b_{1}^{\prime}, b_{2}^{\prime}, c_{1}^{\prime \prime}, c_{2}^{\prime \prime} \\
\left.e_{1}, e_{2}, h_{1}, h_{1}^{\prime}, h_{1}^{\prime \prime} x, y, z\right]
\end{array}\right. \\
& =\frac{1}{\Gamma\left(a_{1}^{\prime}\right) \Gamma\left(a_{2}^{\prime}\right) \Gamma\left(b_{1}^{\prime}\right) \Gamma\left(b_{2}^{\prime}\right)} \int_{0}^{\infty} \cdots \int_{0}^{\infty} e^{-\left(\xi_{1}+\xi_{2}+\xi_{3}+\xi_{4}\right)}
\end{aligned}
$$

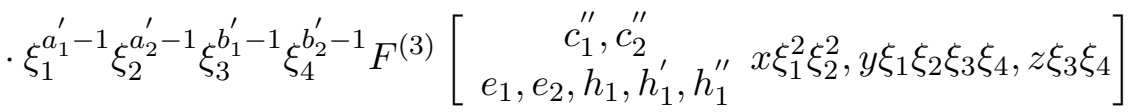

$$
\begin{aligned}
& d \xi_{1} \cdots d \xi_{4}\left(\Re\left(a_{1}^{\prime}\right)>0, \Re\left(a_{2}^{\prime}\right)>0, \Re\left(b_{1}^{\prime}\right)>0, \Re\left(b_{2}^{\prime}\right)>0\right) ;
\end{aligned}
$$

$$
\begin{aligned}
\mathcal{G}_{14}^{(3)} & {\left[\begin{array}{c}
a_{1}^{\prime}, a_{2}^{\prime}, b_{1}^{\prime}, b_{2}^{\prime}, c_{1}^{\prime \prime}, c_{2}^{\prime \prime} \\
\left.g_{1}, g_{2}, h_{1}, h_{1}^{\prime}, h_{1}^{\prime \prime}, h_{2}^{\prime \prime}, h_{3}^{\prime \prime} x, y, z\right]
\end{array}\right.} \\
= & \frac{1}{\Gamma\left(a_{1}^{\prime}\right) \Gamma\left(a_{2}^{\prime}\right) \Gamma\left(b_{1}^{\prime}\right) \Gamma\left(b_{2}^{\prime}\right)} \int_{0}^{\infty} \cdots \int_{0}^{\infty} e^{-\left(\xi_{1}+\xi_{2}+\xi_{3}+\xi_{4}\right)} \\
& \xi_{1}^{a_{1}^{\prime}-1} \xi_{2}^{a_{2}^{\prime}-1} \xi_{3}^{b_{1}^{\prime}-1} \xi_{4}^{b_{2}^{\prime}-1} \cdot F_{2: 1 ; 1}^{0: 0 ; 0}\left[\begin{array}{ccc}
-: & -; & -; \\
g_{1}, g_{2}: & h_{1} ; \quad h_{1}^{\prime} ; & \left.x \xi_{1}^{2} \xi_{2}^{2}, y \xi_{1} \xi_{2} \xi_{3} \xi_{4}\right]
\end{array}\right. \\
& { }_{2} F_{3}\left(c_{1}^{\prime \prime}, c_{2}^{\prime \prime} ; h_{1}^{\prime \prime}, h_{2}^{\prime \prime}, h_{3}^{\prime \prime} ; z \xi_{3} \xi_{4}\right) d \xi_{1} \cdots d \xi_{4} \\
& \left(\Re\left(a_{1}^{\prime}\right)>0, \Re\left(a_{2}^{\prime}\right)>0, \Re\left(b_{1}^{\prime}\right)>0, \Re\left(b_{2}^{\prime}\right)>0\right) ;
\end{aligned}
$$


(2.20)

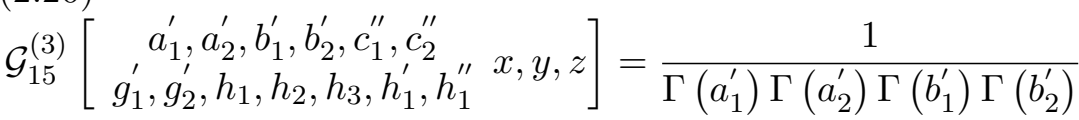

$$
\begin{aligned}
& \cdot \int_{0}^{\infty} \cdots \int_{0}^{\infty} e^{-\left(\xi_{1}+\xi_{2}+\xi_{3}+\xi_{4}\right)} \xi_{1}^{a_{1}^{\prime}-1} \xi_{2}^{a_{2}^{\prime}-1} \xi_{3}^{b_{1}^{\prime}-1} \xi_{4}^{b_{2}^{\prime}-1} \\
& \cdot{ }_{0} F_{3}\left(h_{1}, h_{2}, h_{3} ; x \xi_{1}^{2} \xi_{2}^{2}\right) F_{2: 1 ; 1}^{0: 0 ; 2}\left[\begin{array}{rrr}
-: & -; & c_{1}^{\prime \prime}, c_{2}^{\prime \prime} ; \\
g_{1}^{\prime}, g_{2}^{\prime}: & h_{1}^{\prime} ; & h_{1}^{\prime \prime} ;
\end{array} \xi_{1} \xi_{2} \xi_{3} \xi_{4}, z \xi_{3} \xi_{4}\right] \\
& d \xi_{1} \cdots d \xi_{4}\left(\Re\left(a_{1}^{\prime}\right)>0, \Re\left(a_{2}^{\prime}\right)>0, \Re\left(b_{1}^{\prime}\right)>0, \Re\left(b_{2}^{\prime}\right)>0\right) ;
\end{aligned}
$$

$$
\begin{aligned}
& \mathcal{G}_{16}^{(3)}\left[\begin{array}{c}
a_{1}^{\prime}, a_{2}^{\prime}, b_{1}^{\prime}, b_{2}^{\prime}, c_{1}^{\prime \prime}, c_{2}^{\prime \prime} \\
\left.g_{1}^{\prime \prime}, g_{2}^{\prime \prime}, h_{1}, h_{1}^{\prime}, h_{2}^{\prime}, h_{3}^{\prime}, h_{1}^{\prime \prime} x, y, z\right]
\end{array}\right] \\
& =\frac{1}{\Gamma\left(a_{1}^{\prime}\right) \Gamma\left(a_{2}^{\prime}\right) \Gamma\left(b_{1}^{\prime}\right) \Gamma\left(b_{2}^{\prime}\right)} \int_{0}^{\infty} \cdots \int_{0}^{\infty} e^{-\left(\xi_{1}+\xi_{2}+\xi_{3}+\xi_{4}\right)} \\
& \xi_{1}^{a_{1}^{\prime}-1} \xi_{2}^{a_{2}^{\prime}-1} \xi_{3}^{b_{1}^{\prime}-1} \xi_{4}^{b_{2}^{\prime}-1} \cdot F_{2: 1 ; 1}^{0: 0 ; 2}\left[\begin{array}{rrr}
-: & -; & c_{1}^{\prime \prime}, c_{2}^{\prime \prime} ; \\
g_{1}^{\prime \prime}, g_{2}^{\prime \prime}: & h_{1} ; & h_{1}^{\prime \prime} ;
\end{array} \xi_{1}^{2} \xi_{2}^{2}, z \xi_{3} \xi_{4}\right] \\
& { }_{0} F_{3}\left(h_{1}^{\prime}, h_{2}^{\prime}, h_{3}^{\prime} ; y \xi_{1} \xi_{2} \xi_{3} \xi_{4}\right) d \xi_{1} \cdots d \xi_{4} \\
& \left(\Re\left(a_{1}^{\prime}\right)>0, \Re\left(a_{2}^{\prime}\right)>0, \Re\left(b_{1}^{\prime}\right)>0, \Re\left(b_{2}^{\prime}\right)>0\right) ;
\end{aligned}
$$

$$
\begin{aligned}
\mathcal{G}_{17}^{(3)}\left[\begin{array}{c}
a_{1}^{\prime}, a_{2}^{\prime}, b_{1}^{\prime}, b_{2}^{\prime}, c_{1}^{\prime \prime}, c_{2}^{\prime \prime} \\
\left.h_{1}, h_{2}, h_{3}, h_{1}^{\prime}, h_{2}^{\prime}, h_{3}^{\prime}, h_{1}^{\prime \prime}, h_{2}^{\prime \prime}, h_{3}^{\prime \prime} x, y, z\right]
\end{array}\right. \\
=\frac{1}{\Gamma\left(a_{1}^{\prime}\right) \Gamma\left(a_{2}^{\prime}\right) \Gamma\left(b_{1}^{\prime}\right) \Gamma\left(b_{2}^{\prime}\right)} \int_{0}^{\infty} \cdots \int_{0}^{\infty} e^{-\left(\xi_{1}+\xi_{2}+\xi_{3}+\xi_{4}\right)} \\
\quad \xi_{1}^{a_{1}^{\prime}-1} \xi_{2}^{a_{2}^{\prime}-1} \xi_{3}^{b_{1}^{\prime}-1} \xi_{4}^{b_{2}^{\prime}-1} \cdot{ }_{0} F_{3}\left(h_{1}, h_{2}, h_{3} ; x \xi_{1}^{2} \xi_{2}^{2}\right)_{0} F_{3}\left(h_{1}^{\prime}, h_{2}^{\prime}, h_{3}^{\prime} ; y \xi_{1} \xi_{2} \xi_{3} \xi_{4}\right) \\
\quad{ }_{2} F_{3}\left(c_{1}^{\prime \prime}, c_{2}^{\prime \prime} ; h_{1}^{\prime \prime}, h_{2}^{\prime \prime}, h_{3}^{\prime \prime} ; z \xi_{3} \xi_{4}\right) d \xi_{1} \cdots d \xi_{4} \\
\quad\left(\Re\left(a_{1}^{\prime}\right)>0, \Re\left(a_{2}^{\prime}\right)>0, \Re\left(b_{1}^{\prime}\right)>0, \Re\left(b_{2}^{\prime}\right)>0\right) ;
\end{aligned}
$$




$$
\begin{aligned}
& \mathcal{G}_{18}^{(3)} {\left[\begin{array}{c}
a_{1}^{\prime}, a_{2}^{\prime}, c_{1}^{\prime}, c_{2}^{\prime}, c_{1}^{\prime \prime}, c_{2}^{\prime \prime}, c_{3}^{\prime \prime}, c_{4}^{\prime \prime} \\
e_{1}, e_{2}, h_{1}, h_{1}^{\prime}, h_{1}^{\prime \prime}
\end{array}, y, z\right]=\frac{1}{\Gamma\left(a_{1}^{\prime}\right) \Gamma\left(a_{2}^{\prime}\right)} \int_{0}^{\infty} \int_{0}^{\infty} e^{-\left(\xi_{1}+\xi_{2}\right)} } \\
& \xi_{1}^{a_{1}^{\prime}-1} \xi_{2}^{a_{2}^{\prime}-1} \cdot F^{(3)}\left[\begin{array}{c}
c_{1}^{\prime}, c_{2}^{\prime}, c_{1}^{\prime \prime}, c_{2}^{\prime \prime}, c_{3}^{\prime \prime}, c_{4}^{\prime \prime} \\
e_{1}, e_{2}, h_{1}, h_{1}^{\prime}, h_{1}^{\prime \prime}
\end{array} x \xi_{1}^{2} \xi_{2}^{2}, y \xi_{1} \xi_{2}, z\right] d \xi_{1} d \xi_{2} \\
&\left(\Re\left(a_{1}^{\prime}\right)>0, \Re\left(a_{2}^{\prime}\right)>0\right),
\end{aligned}
$$

where

$$
\begin{aligned}
& F^{(3)}\left[\begin{array}{c}
c_{1}^{\prime}, c_{2}^{\prime}, c_{1}^{\prime \prime}, c_{2}^{\prime \prime}, c_{3}^{\prime \prime}, c_{4}^{\prime \prime} \\
e_{1}, e_{2}, h_{1}, h_{1}^{\prime}, h_{1}^{\prime \prime}
\end{array} x, y, z\right] \\
& =\sum_{i, j, k=0}^{\infty} \frac{\left(c_{1}^{\prime}\right)_{j}\left(c_{2}^{\prime}\right)_{j}\left(c_{1}^{\prime \prime}\right)_{k}\left(c_{2}^{\prime \prime}\right)_{k}\left(c_{3}^{\prime \prime}\right)_{k}\left(c_{4}^{\prime \prime}\right)_{k}}{\left(e_{1}\right)_{i+j+k}\left(e_{2}\right)_{i+j+k}\left(h_{1}\right)_{i}\left(h_{1}^{\prime}\right)_{j}\left(h_{1}^{\prime \prime}\right)_{k} i ! j ! k !} x^{i} y^{j} z^{k} ; \\
& \mathcal{G}_{19}^{(3)}\left[\begin{array}{l}
a_{1}^{\prime}, a_{2}^{\prime}, c_{1}^{\prime}, c_{2}^{\prime}, c_{1}^{\prime \prime}, c_{2}^{\prime \prime}, c_{3}^{\prime \prime}, c_{4}^{\prime \prime} \\
g_{1}^{\prime}, g_{2}^{\prime}, h_{1}, h_{2}, h_{3}, h_{1}^{\prime}, h_{1}^{\prime \prime}
\end{array} \quad x, y, z\right] \\
& =\frac{1}{\Gamma\left(a_{1}^{\prime}\right) \Gamma\left(a_{2}^{\prime}\right)} \int_{0}^{\infty} \int_{0}^{\infty} e^{-\left(\xi_{1}+\xi_{2}\right)} \xi_{1}^{a_{1}^{\prime}-1} \xi_{2}^{a_{2}^{\prime}-1}{ }_{0} F_{3}\left(h_{1}, h_{2}, h_{3} ; x \xi_{1}^{2} \xi_{2}^{2}\right)
\end{aligned}
$$

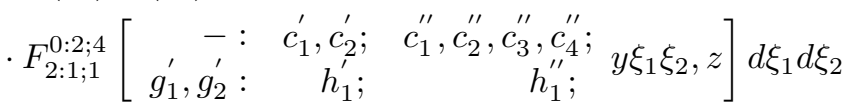

$$
\begin{aligned}
& \left(\Re\left(a_{1}^{\prime}\right)>0, \Re\left(a_{2}^{\prime}\right)>0\right) \text {; }
\end{aligned}
$$

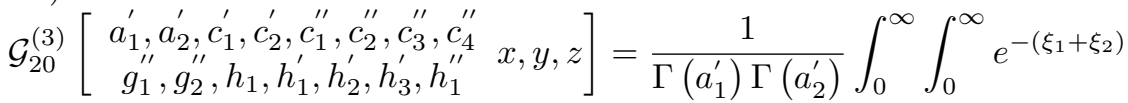

$$
\begin{aligned}
& \xi_{1}^{a_{1}^{\prime}-1} \xi_{2}^{a_{2}^{\prime}-1} \cdot F_{2: 1 ; 1}^{0: 0 ; 4}\left[\begin{array}{rcc}
-: & -; & c_{1}^{\prime \prime}, c_{2}^{\prime \prime}, c_{3}^{\prime \prime}, c_{4}^{\prime \prime} ; \\
g_{1}^{\prime \prime}, g_{2}^{\prime \prime}: & h_{1} ; & \left.h_{1}^{2} \xi_{2}^{2}, z\right]
\end{array}\right] \\
& { }_{2} F_{3}\left(c_{1}^{\prime}, c_{2}^{\prime} ; h_{1}^{\prime}, h_{2}^{\prime}, h_{3}^{\prime} ; y \xi_{1} \xi_{2}\right) d \xi_{1} d \xi_{2}\left(\Re\left(a_{1}^{\prime}\right)>0, \Re\left(a_{2}^{\prime}\right)>0\right),
\end{aligned}
$$

where ${ }_{p} F_{q}$ is the generalized hypergeometric function and $F_{p: q ; k}^{m: n ; r}$ is the Kampé de Fériet hypergeometric function.

\section{Acknowledgements}

The authors should express their deep gratitude for the anonymous reviewers's detailed and helpful comments on this paper. This paper was supported by the Basic Science Research Program through the National Research Foundation of the Republic of Korea funded by the Ministry of Education, Science and Technology (2011-0005224). 


\section{References}

[1] L. Bers, Mathematical Aspects of Subsonic and Transonic Gas Dynamics, Wiley, New York, 1958.

[2] A. Erdélyi, W. Magnus, F. Oberhettinger and F. G. Tricomi, Higher Transcendental Functions, Vol. 1, McGraw-Hill Book Company, New York, Toronto and London, 1953.

[3] H. Exton, Hypergeometric functions of three variables, J. Indian Acad. Math. 4 (1982), 113-119.

[4] F.I. Frankl, Selected Works in Gas Dynamics, Nauka, Moscow, 1973

[5] G. Lohofer, Theory of an electromagnetically deviated metal sphere. 1: Absorbed power, SIAM J. Appl. Math. 49 (1989), 567-581.

[6] A.W. Niukkanen, Generalized hypergeometric series arising in physical and quantum chemical applications, J. Phys. A: Math. Gen. 16 (1983), 1813-1825.

[7] H. M. Srivastava, Hypergeometric functions of three variables, Ganita 15(2) (1964), 97-108.

[8] H. M. Srivastava, Some integrals representing hypergeometric functions, Rend. Circ. Mat. Palermo 16(2) (1967), 99-115.

[9] H. M. Srivastava and J. Choi, Series Associated with the Zeta and Related Functions, Kluwer Academic Publishers, Dordrecht, Boston and London, 2001.

[10] H. M. Srivastava and P. W. Karlsson, Multiple Gaussian Hypergeometric Series, Halsted Press (Ellis Horwood Limited, Chichester), Wiley, New York, Chichester, Brisbane, and Toronto, 1985.

Junesang Choi

Department of Mathematics, Dongguk University, Gyeongju 780-714, Korea.

E-mail: junesang@mail.dongguk.ac.kr

Anvar Hasanov

Department of Mathematics, Dongguk University,

Gyeongju 780-714, Korea.

E-mail: ahasanov@dongguk.ac.kr

Mamasali Turaev

Department of Mathematics, Dongguk University, Gyeongju 780-714, Korea.

E-mail:mturaev@dongguk.ac.kr 